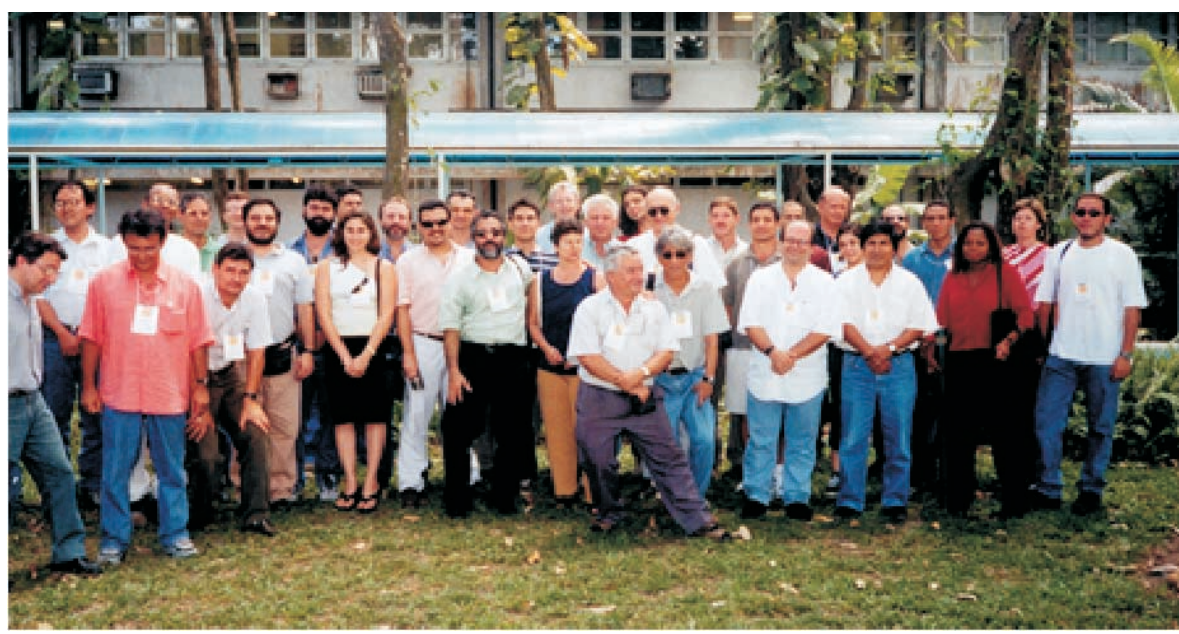

\title{
First Workshop on Materials, Mechanisms and Applications of Superconductivity, and V Escola Brasileira de Supercondutividade
}

The First Workshop on Materials, Mechanisms and Applications of Superconductivity (IWMMAS), and the V Escola Brasileira de Supercondutividade (VEBS), took place at the Departamento de Física, Universidade Federal de Pernambuco, in Recife, Brazil, from 10 to 14 December, 2001. The main objective was to foster the activities in the exciting research field of superconductivity in Brazil, as part of a preliminary program for the forthcoming Seventh International Conference on Materials and Mechanisms of Superconductivity and High Temperature Superconductors, M2S-HTSC-VII, planned to take place in Rio de Janeiro, Brazil, in 2003.

About eighty researchers, including undergraduate and graduate students, attended the IWMMAS and the VEBS, and discussed the state of art in superconductivity, both theoretically and experimentally. The scientific program included basic mechanisms of superconductivity, vortex dynamics, materials preparation and characterization, and applications of superconductivity. The attendees had the chance to interact with leading scientists and to learn many new exciting ideas in the field, both from lectures and also from informal discussions that were made possible by the nice and friendly atmosphere of the meeting. It has been a productive and fruitful event, with the generation of new ideas for the Brazilian superconductivity community.

We are pleased that most of the active Brazilian researchers in the field of superconductivity participated in the IWMMAS, and thankful to the international lectures, Dr. Francisco de la Cruz (Centro Atomico Bariloche, Bariloche, Argentina), Dr. Ernst Helmut Brandt (Max-Planck-Institut für Metallforschung, Stuttgart, Germany), and Prof. Victor V. Moshchalkov (Katholieke Universiteit, Leuven, Belgium).

The papers of this special issue of the Braz. J. Phys. give a broad view of the present time research in superconductivity in Brazil. We thank all authors who kindly spent their time to write these interesting articles in order to register the occurrence of the event. We are specially grateful to Prof. Silvio Salinas, editor of the Braz. J. Phys., for his encouragement and support.

J. Albino Aguiar

Mauro M. Doria

Guest Editors 